\title{
Knee Joint Tendons
}

National Cancer Institute

\section{Source}

National Cancer Institute. Knee Joint Tendons. NCI Thesaurus. Code C161388.

The tendons that connect the quadriceps muscles to the kneecap, the hamstring

muscles to the shin bone and fibula, and the patella to the top part of the fibula, enabling flexion, extension, and slight rotation of the knee. 